\title{
Evaluación de la calidad higiénico sanitaria y de composición de leche de cabra en un rebaño de la raza Saanen
}

\section{Evaluation of hygienic sanitary and composition quality of goat milk in a Saanen breed herd}

\author{
Grille, Lucía ${ }^{(1)}$, Carro, Silvana ${ }^{(1)}$, Escobar, Daniela ${ }^{(4)}$, Bentancor, Lorena ${ }^{(3)}$, Borges, Alejandra ${ }^{(4)}$, Cruz, Daniel ${ }^{(3)}$, \\ González, Silvana ${ }^{(2)}$
}

${ }^{(1)}$ Departamento de Ciencia y Tecnología de la Leche, Facultad de Veterinaria, UdelaR, Montevideo, Uruguay - (2) Parque de Actividades Agroindustriales (PAGRO), Unidad Montevideo Rural, Intendencia de Montevideo, Uruguay - (3) Facultad de Veterinaria, UdelaR, Montevideo, Uruguay (trabajo final de grado) - ${ }^{(4)}$ Gerencia de I+D+I, Laboratorio Tecnológico del Uruguay, LATU, Uruguay.

Contacto: lgrille@gmail.com

Recibido: 4/6/2013 - Aprobado: 25/11/2013

\begin{abstract}
$\underline{\text { Resumen }}$
Uruguay es un país lechero y sus reglamentaciones son referidas principalmente a leche bovina. La leche de cabra es un alimento completo, recomendado para niños y adultos mayores, pero su estudio ha sido escaso en el país. Se estudió la calidad higiénico-sanitaria y de composición de leche caprina en un rebaño de raza Saanen durante un ciclo de lactancia. Se utilizaron 25 animales y se obtuvieron muestras de tanque quincenalmente. Se analizaron recuento de mesófilos aerobios totales (RMAT), coliformes totales (CT) y Staphylococcus coagulasa positiva (SCP), recuento de células somáticas (RCS), composición, ácidos grasos, acidez Dörnic, pH y densidad. Los resultados fueron: RMAT de 3,85 $\pm 0,69$ ufc/ml $\log _{10}, R_{C S} 6,91 \pm .0,55 \mathrm{cel} / \mathrm{ml} \mathrm{Log}_{10}, \mathrm{CT}$ $2,47 \pm 0,84 \mathrm{ufc} / \mathrm{ml} \log _{10}$, SCP 1,13 $\pm 0,29 \mathrm{ufc} / \mathrm{ml} \mathrm{Log}_{10}$. Se obtuvieron valores de grasa: 3,58 $\pm 0,69 \%$, proteína: $2,71 \pm 0,07 \%$, lactosa: $3,84 \pm 0,10 \%$, acidez: $14,19 \pm 0,39{ }^{\circ} \mathrm{D}, \mathrm{pH}: 6,66 \pm 0,06$ y densidad: $1,026 \pm 0,26 \mathrm{~g} / \mathrm{ml}$, concordantes con trabajos realizados en animales sanos para la misma raza. Los ácidos grasos de cadena corta (caproico, caprílico, cáprico) representan un $12 \%$ de los ácidos grasos totales, valores superiores a la leche de vaca $(\approx \mathbf{5 \%})$. Los resultados obtenidos son útiles para una futura reglamentación en leche de cabra. Por sus diferencias con la de vaca es importante generar estándares específicos.

Palabras clave: Cabra, leche, composición, ácidos grasos, calidad.
\end{abstract}

\begin{abstract}
$\underline{\text { Abstract }}$
Uruguay is a dairy country where predominates regulations in bovine milk. Goat milk is a complete food, suitable for children and seniors, but there are few studies in our country. We studied the hygienic-sanitary quality and composition of milk in Saanen goats during a complete lactation. 25 animals were used. Samples were obtained from the milk tank every two weeks. Total aerobic mesophilic count (TAMC), coliforms and Staphylococcus coagulase positive, Dörnic acidity, pH, composition, fatty acids and Somatic Cell Count (SCC) were analized. The results were: TAMC: $3,85 \pm 0,69 \mathrm{ufc} / \mathrm{ml} \mathrm{Log}_{10}, \mathrm{SCC} 6,91 \pm .0,55 \mathrm{cel} / \mathrm{ml}$

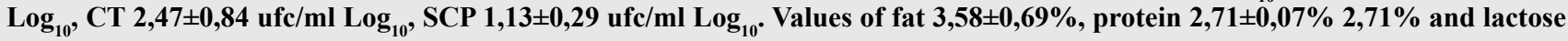
$3,84 \pm 0,10 \%$, acidity $14,19 \pm 0,39{ }^{\circ} \mathrm{D}, \mathrm{pH} 6,66 \pm 0,06$ and density $1,026 \pm 0,26 \mathrm{~g} / \mathrm{ml}$ were obtained, being consistent with work performed in healthy animals and reported for the same breed. Caproic, caprylic and capric acids account for a $12 \%$ of the total fatty acids, higher than the cow milk $(\approx 5 \%)$.

The values obtained are a useful basis for future goat milk regulation. Considering its difference with cow's milk specific standards are necessary.

Keywords: Goat, milk, composition, fatty acids, quality.
\end{abstract}

\section{Introducción}

\section{Importancia en la nutrición y salud humana}

La imagen de la leche de cabra se basa en la salud y el valor nutricional. Estos aspectos positivos pueden ser beneficiosos para los productores e industriales siempre que se mantengan estrictas condiciones microbiológicas. Al igual que la de vaca, la leche de cabra no puede sustituir la materna, sin embargo podría ser utilizada en humanos con efectos beneficiosos asociados con otros alimentos después del año de edad (Desjeux, 1993). De acuerdo con Haenlein (2004), existen tres razones para la demanda de leche de cabra, la primera es el consumo doméstico, demanda en aumento debido al crecimiento de la población humana. La segunda razón es el interés en los subproductos, especialmente quesos y yogures, en muchos países desarrollados. La tercera razón se deriva de un aspecto 
médico, relativo a las alergias a la leche de vaca y otros problemas gastrointestinales. Los triglicéridos de cadena media, MCT (entre 6 y 10 átomos de carbono, según estos autores), presentan un interés muy particular desde un punto de vista terapéutico, a causa de su utilidad en el tratamiento de ciertas enfermedades metabólicas (Boza y Sanz, 1997; Sanz et al., 2003). También son beneficiosos para los síndromes de mala absorción, mejora de la digestión y trastornos del sueño, especialmente en los niños (Haenlein, 2001), y de enfermedades coronarias, fibrosis quística y cálculos biliares por su capacidad única de proporcionar energía metabólica mientras que reduce, inhibe y disuelve los depósitos de colesterol (Park, 1994; Sanz et al., 2003).

El bajo peso molecular e hidrosolubilidad de los MCT facilita la acción de las enzimas digestivas, haciendo que su hidrólisis sea más rápida y completa que la de los triglicéridos de cadena larga. A diferencia de éstos, la digestión de los MCT comienza a producirse en el estómago, ya que la lipasa gástrica prácticamente sin acción sobre los triglicéridos de cadena larga inicia la hidrólisis de los MCT, la cual será completada por la lipasa pancreática a un ritmo cinco veces superior a la hidrólisis de los triglicéridos de cadena larga (García, 1996 citado por Sanz et al., 2003).

Otra cualidad destacada de la leche de pequeños rumiantes son sus efectos beneficiosos y terapéuticos en las personas y especialmente en los niños que tienen alergia a la leche de vaca (Ribeiro y Ribeiro, 2010; Haenlein, 2001; Park, 1994). Park (1994) encontró que aproximadamente el $40 \%$ de todos los pacientes que son sensibles a la proteínas lácteas de la vaca toleran las de la cabra y que es muy útil para las personas que sufren de problemas como acidez, eczema, asma, migraña, colitis, úlcera estomacal, trastornos digestivos, enfermedades del hígado y de la vesícula biliar y síntomas relacionados con el estrés, tales como insomnio e indigestión neurótica.

\section{Características de la leche de cabra}

Según Larrosa y Kremer (1990), esta leche recién ordeñada presenta un sabor dulzón y olor neutro. Sin embargo, Boza y Sanz (1997) mencionan que su olor es fuerte como consecuencia de la absorción de compuestos aromáticos durante su manejo, generalmente inadecuado. Estos autores coinciden en que el color es más blanco que el de vaca, lo que se debe a la diferencia en el contenido de carotenos. Park et al. (2007), citando a varios autores, indican que la leche de cabra posee un contenido de sólidos totales y de nutrientes en una posición intermedia entre la leche de vaca y oveja. Se diferencia de la leche de vaca y humana en la digestibilidad más alta, alcalinidad, capacidad buffer y en sus características terapéuticas para la salud y nutrición humanas (Jandal, 1996; Park et al., 2007). La digestibilidad es más alta en la leche de cabra que en la de vaca debido a la homogeneización natural, ya que los glóbulos de grasa de la leche caprina son más pequeños (promedio de $3,49 \mu \mathrm{m}$ frente a $4,55 \mu \mathrm{m}$ en los de leche de vaca) y tienen una superficie mayor para el ataque de las lipasas intestinales, lo cual mejora la digestibilidad y la eficiencia del metabolismo de los lípidos (Park, 1994; Jandal, 1996; Park et al., 2007; De Souza et al., 2009).

De acuerdo a Morand-Fehr et al. (2007), la composición de macro y micronutrientes en la leche de cabra depende de los principales factores de producción que constituyen el sistema productivo: genotipo, características reproductivas y sanitarias de los animales, condiciones agro-climáticas y ambiente socioeconómico; así como de los métodos de producción: alimentación y ordeño. Las proteínas de esta leche no tienen grandes diferencias en comparación con la leche de vaca, excepto en la digestibilidad y en la secuencia de ciertas variantes genéticas (Desjeux, 1993). Los lípidos son los componentes más importantes de la leche en términos de costo, nutrición y en las características físicas y sensoriales que imparten a los productos lácteos. Los triglicéridos son el grupo más importante (casi 98\%), incluyendo un gran número de ácidos grasos esterificados (Park et al., 2007). Los ácidos grasos de cadena media (MCT) son triglicéridos formados por ácidos grasos cuya cadena carbonada tiene entre 6 y 14 átomos de carbono, y alcanzan normalmente un porcentaje mayor del $30 \%$, a diferencia de la leche de vaca que no alcanza más del $20 \%$ (Sanz et al., 2003). Es por esto por lo que los ácidos grasos caproico (C6:0), caprílico (C8:0) y cáprico (C10:0) toman su nombre concretamente de la leche en donde aparecen mayormente y en la cual estos ácidos alcanzan un $15 \%$, valor que solo llega al $5 \%$ en la leche de vaca (Boza y Sanz, 1997). Estos niveles elevados de ácido butírico, caproico, caprílico y cáprico le confieren características únicas para fabricar quesos, ya que intervienen en su sabor (Draksler et al., 2002).

La lactosa es el principal carbohidrato de la leche y es un valioso nutriente que favorece la absorción intestinal del calcio, magnesio y fósforo, y la utilización de la vitamina D. Es importante para el mantenimiento del equilibrio osmótico entre el torrente sanguíneo y las células alveolares de la glándula mamaria durante la síntesis de leche, también para la secreción en la luz alveolar y en el sistema de conductos de la ubre (Park et al., 2007).

La calidad higiénico-sanitaria puede ser avalada en base a dos indicadores: el recuento de células somáticas, que indica la frecuencia de animales con mastitis en el rebaño, y el recuento total de bacterias, que refleja las condiciones de higiene y almacenamiento de la leche desde su obtención hasta el envío a la industria (De Souza et al., 2009). Según Zweifel et al. (2005), la evaluación de la calidad microbiológica es importante en la inocuidad alimentaria y, por tanto, en la protección de la salud de los consumidores. En la Unión Europea el recuento total de mesófilos aerobios (RTMA) en leche cruda de pequeños rumiantes, usada para el consumo de leche fluida y subproductos y tratada térmicamente, no debe superar el valor de $6,0 \mathrm{ufc} / \mathrm{ml} \mathrm{Log}_{10}$, en cambio, si esa leche cruda se destina para la producción de productos sin tratamiento térmico no debe exceder a 5,69 ufc/ml $\log _{10}$. En Brasil uno de los requisitos para que la leche de cabra sea de buena calidad, además de los tenores mínimos de los principales componentes, es el RTMA, el cual no puede superar a $5,69 \mathrm{ufc} / \mathrm{ml} \mathrm{Log}_{10}$ para la leche cruda (De Souza et al., 2009).

Las células somáticas son las células blancas de la sangre (leucocitos) y constituyen la defensa contra aquellas bacterias que penetran la barrera física del canal del pezón de la ubre. Además, se encuentran células epiteliales provenientes de la descamación del epitelio de la glándula mamaria (De Souza et al., 2009). En las cabras el recuento de células somáticas (RCS) presenta algunas particularidades, ya que en su secreción de leche se eliminan partículas citoplasmáticas junto a los leucocitos y células epiteliales. Estas partículas son no nucleadas, pero similares a los leucocitos en el diámetro y morfología (Gomes et al., 2006). De acuerdo con Paape et al. (2007), el RCS se ha convertido en un parámetro aceptado para evaluar la calidad de leche y es la base de los programas de control mundiales de leche de vaca, oveja y cabra para prevenir el ingreso de la leche anormal o no apta para el consumo humano. No obstante, Robertson y Muller (2005) sostienen que en cabras es más difícil conectar el RCS con una posible infección intramamaria. La razón de la controversia que existe entre RCS y la infección por mastitis es que la secreción de la leche de cabra difiere de la de vaca. Mientras que en la vaca la secreción es merócrina (la leche es presionada hacia afuera de los alvéolos), en la cabra es apócrina, con la aparición de un gran número de partículas citoplasmáticas en la leche normal. Estas partículas que no contienen ADN enmascaran y complican la interpretación de la respuesta de los leucocitos a la inflamación. Paape et al. (2007) coinciden con Robertson y Muller (2005) al mencionar que en las cabras el RCS no es aplicable para evaluar la calidad de leche debido a que el RCS de cabras sanas es más alto que en vacas y ovejas sanas. Pridalová et al. (2009) indican que el alto valor de RCS de la leche caprina es en parte causado por un aumento en la tasa de desprendimiento de células epiteliales y partículas de citoplasma como consecuencia de la secreción apócrina.

Por todo esto se considera de importancia el estudio de la leche de cabra en Uruguay, donde no existía, hasta el momento de realizar este trabajo, reglamentación que definiera sus parámetros de calidad higiénico-sanitaria ni de composición. Luego de culminado este estudio se establecieron pautas a nivel oficial para esta especie, con base en los resultados hallados. 


\section{Materiales y Métodos}

\section{Animales}

Se utilizaron 25 cabras de la raza Saanen, las cuales se encontraban en el Parque de Actividades Agropecuarias (PAGRO) perteneciente a la Intendencia de Montevideo, localizado en la zona de Colón, Montevideo, Uruguay.

Al tratarse de un sistema semi-extensivo, los animales se alimentaron con ración lechera y praderas implantadas (avena y alfalfa) durante toda la lactancia.

\section{Metodología de muestreo}

Para cumplir con los objetivos planteados se realizaron muestreos para análisis fisicoquímicos y microbiológicos, según la metodología descripta por la Federación Internacional de Lechería (FIL-IDF 50C:1995 citado por Pinto et al., 1998).

Las muestras se tomaron por triplicado con una frecuencia quincenal del volumen producido en el ordeñe matutino ( 2 por mes, exceptuando el primer mes que se realizó 1 muestreo). Este muestreo tuvo lugar durante los meses que duró la lactancia (setiembre de 2010 a febrero de 2011), constituyendo un total de 11 muestreos (M1 a M11). Se tomaron muestras representativas del ordeñe (tanque), para lo cual previamente se mezcló mecánicamente durante por lo menos 5 minutos (hasta obtener una homogeneización adecuada) y luego se extrajo una alícuota del volumen. Las muestras se colocaron en recipientes isotérmicos refrigerados y se mantuvieron en refrigeración $\left(4{ }^{\circ} \mathrm{C}\right)$ durante el transporte hasta su llegada al laboratorio. Los análisis fueron realizados dentro de las 24 horas posteriores a la extracción.

\section{Análisis de laboratorio}

\section{Análisis de composición y fisicoquímico}

Las muestras se analizaron en el laboratorio de calidad de leche del Departamento de Ciencia y Tecnología de la Leche de Facultad de Veterinaria (UdelaR) y en el Laboratorio Tecnológico del Uruguay (LATU). A cada muestra se le realizaron análisis de composición: materia grasa, proteína, lactosa, sólidos no grasos (SNG) y densidad, utilizando un equipo denominado Lactomilk ${ }^{\circledR}$ marca Lactoscan, modelo SLP, origen Bulgaria. El equipo se calibró utilizando los métodos de referencia para cada análisis (materia grasa: Gerber, proteína: Kjeldahl, SNG: gravimétricos). Se realizó recuento de células somáticas (RCS) mediante la técnica de Breed (International Dairy Federation, 1995) determinación de acidez Dörnic (Pinto et al., 1998) y pH mediante método de evaluación potenciométrico, utilizando un modelo de electrodos denominado Oakton ${ }^{\circledR}$. El equipo analizador de leche presenta para cada componente analizado la siguiente precisión: materia grasa: $\pm 0,06 \%$; proteína: $\pm 0,15 \%$; lactosa: $\pm 0,2 \%$; SNG (sólidos no grasos): $\pm 0,15 \%$; densidad: $\pm 0,3 \mathrm{~kg} / \mathrm{m} 3$.

\section{Análisis microbiológicos}

Se realizó recuento de mesófilos aerobios totales (RTMA), coliformes totales y Staphylococcus coagulasa positiva (APHA, 2001).

\section{Análisis de composición de ácidos grasos}

La determinación del análisis de la composición de ácidos grasos de leche de cabra fue realizada mediante cromatografía gaseosa con detector de espectrometría de masa (EI), conforme con el método AOCS Ce 1-62, AOCS Ce 1-91. Para ello se realizó una extracción en frío con distintos solventes, según Norma ISO 14156 (2001), y una metilación de los ácidos grasos conforme método AOCS Ce 2-66, según la misma norma. Los ácidos grasos metilados se analizaron mediante un cromatógrafo de gases (Agilent $6890 \mathrm{~N}$ con detector de masa Agilent 5973 Inert), equipado con una columna capilar (Supelco SP- $2560,100 \mathrm{~m}$ x 0.25 mm ID x $0.20 \mu \mathrm{m}$ film). Se utilizó gas Carrier Helio $99.999 \%$ de pureza. La inyección split fue hecha a una relación 1:10; volumen $1 \mu \mathrm{L}$. Las condiciones adoptadas del equipo fueron: temperatura del inyector $250{ }^{\circ} \mathrm{C}$; temperatura inicial del horno: $170^{\circ} \mathrm{C}$; rampa de temperatura $1{ }^{\circ} \mathrm{C} / \mathrm{min}$; temperatura final del horno $225^{\circ} \mathrm{C}$ durante $40 \mathrm{~min}$; temperatura de la fuente $200{ }^{\circ} \mathrm{C}$, Ionización por impacto electrónico. La identificación y cuantificación de los ácidos grasos se llevó a cabo mediante la comparación de los tiempos de retención relativos de cada pico cromatográfico con los picos obtenidos de la inyección de la solución de FAME de 37 componentes (Supelco 47885-U). Los resultados de los ácidos grasos fueron expresados en porcentaje másico.

\section{Análisis estadístico}

Los datos se analizaron utilizando estadística descriptiva con el programa Microsoft Excel (Office 2010).

\section{Resultados}

A continuación se presentan los resultados obtenidos de calidad higiénico-sanitaria de leche caprina durante un ciclo de lactancia (6 meses).

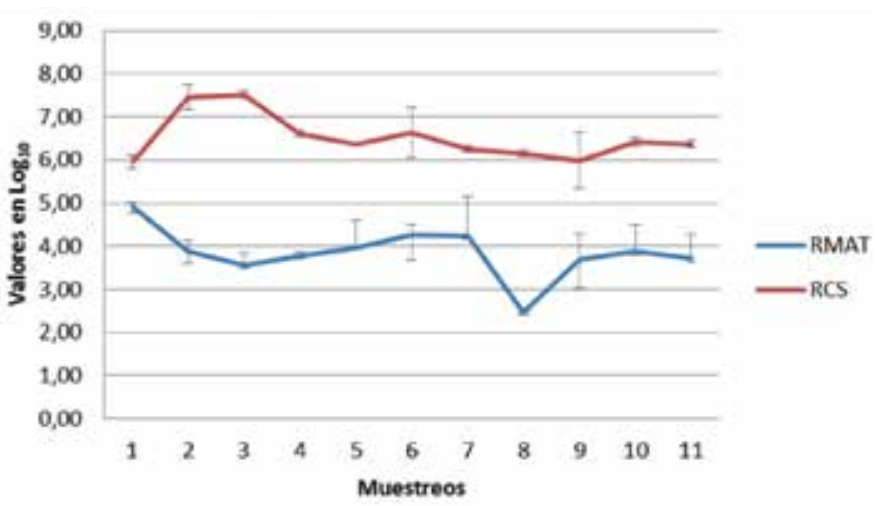

Gráfico 1. Recuento de mesófilos aerobios totales (RMAT) y recuento de células somáticas (RCS) durante un ciclo de lactancia en leche caprina (ufc/ml $\left.\log _{10}\right) \cdot(n=33)$

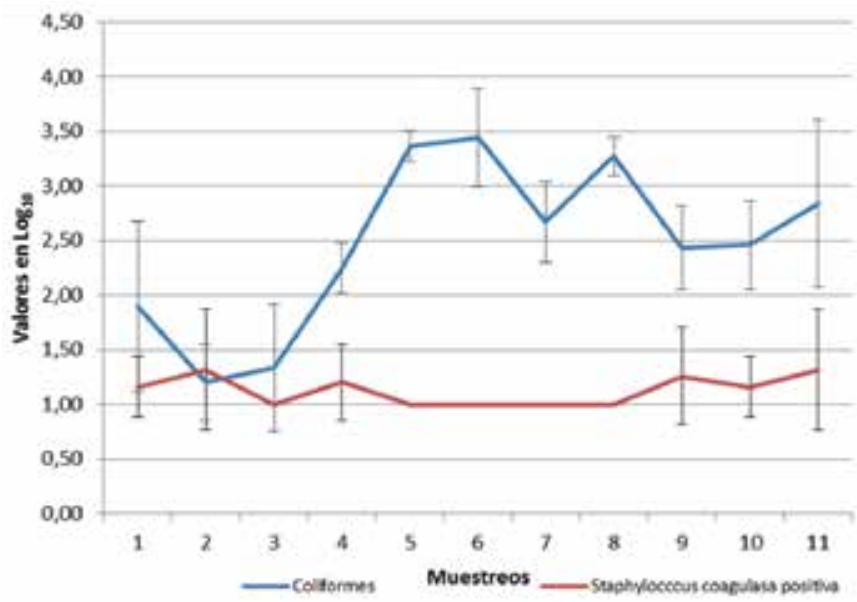

Gráfico 2. Recuento de coliformes totales y Staphylococcus coagulasa positiva durante un ciclo de lactancia en leche caprina (ufc/ml Log $\left.\log _{10}\right)(n=33)$. 
En las Tablas 1 y 2 y Figura 1 se muestran los resultados obtenidos de composición de leche caprina.

\begin{tabular}{|c|c|c|c|c|}
\hline & MG (\%) & $\begin{array}{c}\text { PROTEÍNA } \\
\text { (\%) }\end{array}$ & $\begin{array}{c}\text { LACTOSA } \\
\mathbf{( \% )}\end{array}$ & SNG (\%) \\
\hline M1 & $3,71 \pm 0,02$ & $2,8 \pm 0,01$ & $3,97 \pm 0,02$ & $7,53 \pm 0,03$ \\
\hline M2 & $3,91 \pm 0,04$ & $2,71 \pm 0,02$ & $3,84 \pm 0,03$ & $7,28 \pm 0,06$ \\
\hline M3 & $3,46 \pm 0,18$ & $2,79 \pm 0,02$ & $3,96 \pm 0,04$ & $7,49 \pm 0,05$ \\
\hline M4 & $4,10 \pm 0,08$ & $2,72 \pm 0,00$ & $3,84 \pm 0,05$ & $7,29 \pm 0,05$ \\
\hline M5 & $2,83 \pm 0,01$ & $2,75 \pm 0,03$ & $3,94 \pm 0,01$ & $7,47 \pm 0,03$ \\
\hline M6 & $3,16 \pm 0,07$ & $2,66 \pm 0,05$ & $3,78 \pm 0,01$ & $7,16 \pm 0,02$ \\
\hline M7 & $3,30 \pm 0,16$ & $2,69 \pm 0,02$ & $3,82 \pm 0,02$ & $7,24 \pm 0,04$ \\
\hline M8 & $2,60 \pm 0,02$ & $2,75 \pm 0,11$ & $3,93 \pm 0,03$ & $7,42 \pm 0,06$ \\
\hline M9 & $4,98 \pm 0,05$ & $2,56 \pm 0,01$ & $3,62 \pm 0,01$ & $6,88 \pm 0,01$ \\
\hline M10 & $3,30 \pm 0,05$ & $2,64 \pm 0,01$ & $3,74 \pm 0,01$ & $7,09 \pm 0,03$ \\
\hline M11 & $4,06 \pm 0,05$ & $2,71 \pm 0,01$ & $3,83 \pm 0,03$ & $7,28 \pm 0,03$ \\
\hline Media & $3,58 \pm 0,65$ & $2,71 \pm 0,07$ & $3,84 \pm 0,10$ & $7,28 \pm 0,19$ \\
\hline
\end{tabular}

Tabla 1. Composición de la leche caprina durante un ciclo de lactancia (6 meses) $(n=33)$. La media corresponde a 11 muestreos.

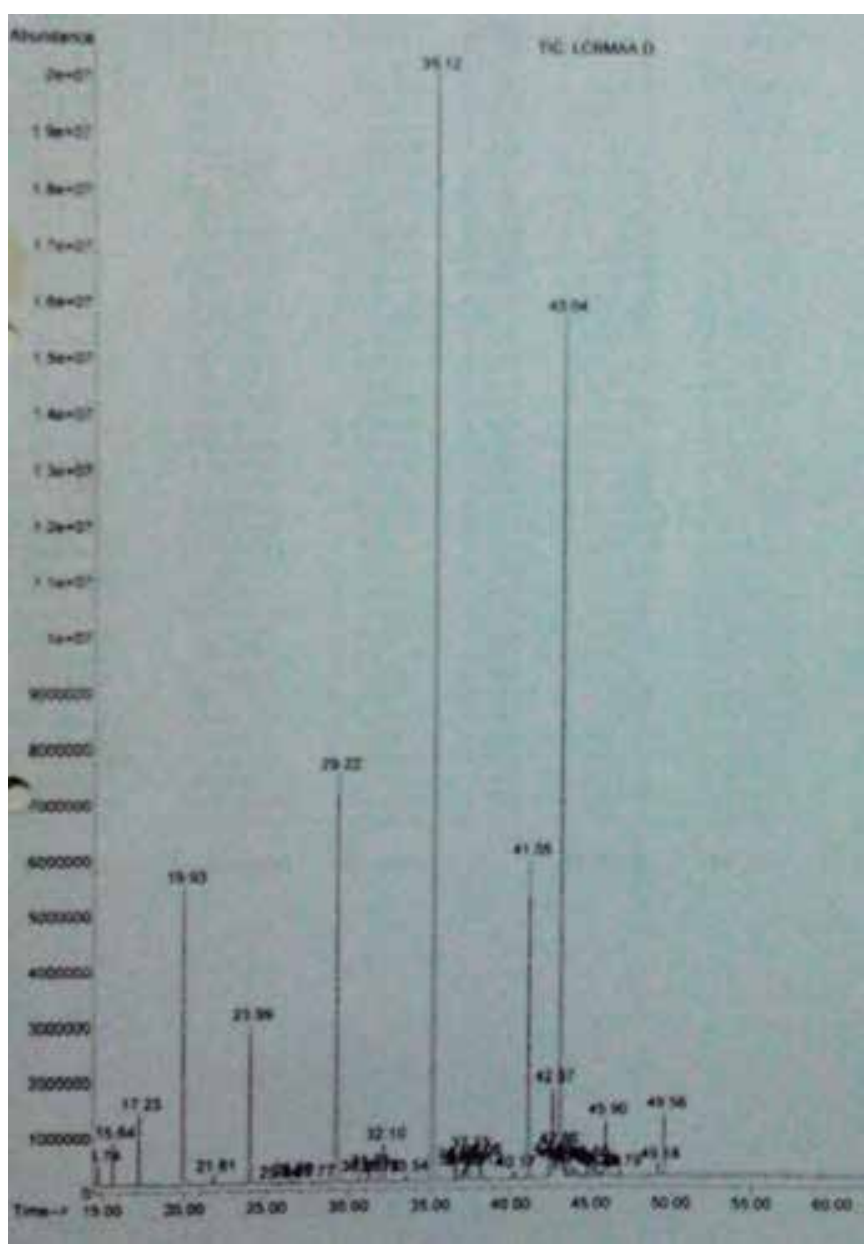

Figura 1. Perfil cromatográfico de ácidos grasos en leche de cabra.

\begin{tabular}{|c|c|}
\hline Ácidos grasos & $\begin{array}{l}\mathrm{g} / 100 \mathrm{~g} \text { total de } \\
\text { ácidos grasos }\end{array}$ \\
\hline C $4: 0$ & 0,56 \\
\hline C 6:0 & 1,28 \\
\hline C 8:0 & 2,05 \\
\hline C $10: 0$ & 8,33 \\
\hline C $10: 1$ & 0,15 \\
\hline C 11:0 & 0,09 \\
\hline C 12:0 & 4,21 \\
\hline C $12: 1$ cis & 0,19 \\
\hline C 13:0 & 0,21 \\
\hline C $14: 0$ & 11,72 \\
\hline C $14: 1$ cis & 0,21 \\
\hline C 15:0 & 1,62 \\
\hline C $15: 1$ & 0,04 \\
\hline C $16: 0$ & 31,09 \\
\hline C 16:1 cis & 0,65 \\
\hline C 16:1 trans & 0,25 \\
\hline C 17:0 & 1,70 \\
\hline C $17: 1$ cis & 0,30 \\
\hline C 18:0 & 9,55 \\
\hline C 18:1 cis & 20,36 \\
\hline C $18: 1$ trans & 2,24 \\
\hline C $18: 2$ cis $(n-6)$ & 1,44 \\
\hline C 18:2 trans $(n-6)$ & 0,55 \\
\hline C 18:2 CLA & 0,83 \\
\hline C 18:3 (n-3) & 0,56 \\
\hline C 19:0 & 0,22 \\
\hline C 19:1 & 0,15 \\
\hline C 20:0 & 0,17 \\
\hline C $20: 1$ cis & 0,05 \\
\hline C $20: 2$ cis (n-6) & 0,04 \\
\hline C $20: 3$ cis $(n-3)$ & 0,06 \\
\hline C 20:4 (n-6) & 0,08 \\
\hline C 20:5 (n-3) & 0,03 \\
\hline C 21:0 & 0,05 \\
\hline C 22:0 & 0,05 \\
\hline C 22:1 cis & 0,04 \\
\hline C $22: 2$ cis & 0,02 \\
\hline C 22:5 (n-6) & 0,06 \\
\hline C 22:6 (n-3) & 0,02 \\
\hline C 23:0 & 0,03 \\
\hline C 24:0 & 0,01 \\
\hline SFA & 2,34 \\
\hline MUFA & 0,70 \\
\hline PUFA & 0,07 \\
\hline GRASA TRANS & 0,10 \\
\hline CLA & 0,03 \\
\hline GRASA TOTAL & 3,22 \\
\hline UFA $(\%)$ & 27,33 \\
\hline SFA (\%) & 72,67 \\
\hline
\end{tabular}

Tabla 2. Perfil de ácidos grasos de leche de cabra de la raza Saanen $(n=11)$. SFA: ácidos grasos saturados; MUFA: ácidos grasos monoinsaturados; PUFA: ácidos grasos poliinsaturados; CLA: ácido linoleico conjugado; \%UFA: porcentaje de ácidos grasos insaturados; \%SFA: porcentaje de ácidos grasos saturados. 
En la Tabla 3 se presentan los resultados de las propiedades fisicoquímicas de las muestras de leche analizadas para este rebaño.

\begin{tabular}{|c|c|c|c|}
\hline & MG (\%) & $\begin{array}{c}\text { PROTEÍNA } \\
\text { (\%) }\end{array}$ & $\begin{array}{c}\text { LACTOSA } \\
\text { (\%) }\end{array}$ \\
\hline M1 & $13,66 \pm 0,55$ & $6,81 \pm 0,02$ & $1,030 \pm 0,00$ \\
\hline M2 & $19,00 \pm 0,22$ & $6,65 \pm 0,01$ & $1,025 \pm 0,00$ \\
\hline M3 & $14,00 \pm 0,28$ & $6,65 \pm 0,04$ & $1,026 \pm 0,57$ \\
\hline M4 & $13,00 \pm 0,00$ & $6,62 \pm 0,01$ & $1,025 \pm 0,00$ \\
\hline M5 & $13,66 \pm 0,57$ & $6,63 \pm 0,01$ & $1,026 \pm 0,00$ \\
\hline M6 & $12,66 \pm 1,10$ & $6,68 \pm 0,05$ & $1,025 \pm 0,00$ \\
\hline M7 & $14,00 \pm 0,00$ & $6,69 \pm 0,00$ & $1,025 \pm 0,00$ \\
\hline M8 & $12,66 \pm 0,57$ & $6,64 \pm 0,05$ & $1,026 \pm 0,00$ \\
\hline M9 & $15,00 \pm 0,00$ & $6,62 \pm 0,02$ & $1,026 \pm 0,57$ \\
\hline M10 & $14,33 \pm 0,57$ & $6,64 \pm 0,02$ & $1,025 \pm 0,00$ \\
\hline M11 & $16,00 \pm 0,00$ & $6,61 \pm 0,00$ & $1,025 \pm 0,57$ \\
\hline
\end{tabular}

Tabla 3. Propiedades fisicoquímicas de la leche caprina durante un ciclo de lactancia $(n=33)$.

\section{Discusión}

Los resultados obtenidos en este trabajo en relación a la calidad higiénica en base al RMAT durante toda la lactación mostraron un

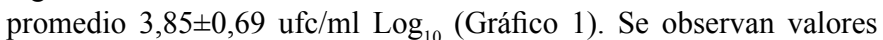
considerablemente inferiores a los encontrados en estudios similares en países como Venezuela y Argentina, donde Cordiviola et al. (2007) obtuvieron valores de $6,33 \mathrm{ufc} / \mathrm{mlLog}_{10}$ y Garcia et al. (2009), en cabras Saanen, de 7,52 ufc/ml $\log _{10}$. También coinciden con Morgan et al. (2003) en Grecia, 7,55 ufc/mL $\log _{10}$ y Portugal, 7,66 ufc/ml $\log _{10}$, y Zweifel et al. (2005) en Suiza, 6,00 ufc/ml $\log _{10}$.

En referencia al recuento de células somáticas en la región, en Brasil Gomes et al. (2006) hallaron valores promedio de 5,41 y 5,93 cél $/ \mathrm{ml} \log _{10}$. Mientras que en Argentina, Cordiviola et al. (2007), utilizando el método de recuento por microscopía directa (Prescott y Breed), encontraron un valor promedio de $6,90 \mathrm{cel} / \mathrm{ml} \log _{10}$ en animales que no presentaban mastitis, lo cual se asemeja a los valores hallados en este estudio aplicando la misma metodología. A su vez, Robertson y Muller (2005) afirman que los valores varían dependiendo del método de análisis, y que estos aumentan si dicho método no distingue células nucleadas de las no nucleadas. En esta investigación se observó un valor promedio de recuento de células somáticas de $6,91 \mathrm{cel} / \mathrm{ml} \log _{10}$ todos animales sanos (Gráfico 1), lo que indicaría que existe una marcada coincidencia con el resto de los autores citados acerca del poco valor relativo del recuento de células somáticas como indicador de infecciones intramamarias en leche caprina.

En cuanto a los microorganismos, los coliformes constituyen el grupo de enterobacterias más importante presente en leche cruda. Se encuentran prácticamente en todas partes, por lo que son utilizados como "indicadores", es decir, microorganismos cuya presencia en gran número delata prácticas de trabajo en malas condiciones higiénicas (Draksler et al., 2002). En relación a coliformes totales para leche cruda de cabra, Bergonier et al. (2009) reportaron valores de 5,92 ufc/ $\mathrm{mlLog}_{10}$; Cordiviola et al. (2007) obtuvieron valores de 5,00 ufc $/ \mathrm{ml}$ $\log _{10}$, y Morgan et al. (2003) hallaron valores promedio de coliformes totales de $6,22 \mathrm{ufc} / \mathrm{ml} \log _{10} 7,60 \mathrm{ufc} / \mathrm{ml} \mathrm{Log}_{10}$ en Grecia y Portugal, respectivamente. En rodeos caprinos en países de la región algunos autores como Chávez et al. (2007) registraron un promedio de 5,00 $\mathrm{ufc} / \mathrm{ml} \log _{10}$. Schmidt et al. (2008) indican que los coliformes totales variaron entre 3,63 y $6,14 \mathrm{ufc} / \mathrm{ml} \log _{10}$. Por otro lado, en Venezuela se observaron valores más elevados; García et al. (2009) reportan un valor promedio de $5,91 \mathrm{ufc} / \mathrm{mlLog}_{10}$. En este estudio los valores de coliformes totales encontrados para este rodeo fueron de $2,47 \pm 0,84$ $\mathrm{ufc} / \mathrm{ml} \log _{10}$, muy inferiores a los citados por la bibliografía.
Los patógenos más prevalentes en la cabra son los estafilococos. Las enterotoxinas estafilocócicas, producidas por Staphylococcus coagulasa positiva, en general se caracterizan por ser termorresistentes y persisten en los productos lácteos elaborados con leche de animales infectados, a pesar de la pasteurización o esterilización, lo que implica un riesgo para el consumidor (Contreras et al., 2010). En cuanto a valores de Staphylococcus coagulasa positiva, Morgan et al. (2003) registraron valores promedio de 5,22 ufc/ml $\log _{10}, 4,87 \mathrm{ufc} / \mathrm{ml}$ $\log _{10}$. En Brasil, Queiroga et al. (2002) indican un valor máximo de Staphylococcus aureus de 3,54 ufc/ml $\log _{10}$. Muehlherr et al. (2003), en Suiza, detectaron Staphylococcus aureus en el 31,7\% de las muestras, con un valor promedio menor a $1,00 \mathrm{ufc} / \mathrm{ml} \log _{10}$ y el valor máximo fue de 4,34 ufc/ml $\log _{10}$. En Uruguay en el Reglamento Bromatológico Nacional (1994) se admiten hasta 3,00 ufc/mL $\log _{10}$ de Staphylococcus aureus en leche cruda bovina, y no existen niveles establecidos para leche caprina. En este trabajo el valor promedio encontrado, de 1,13 $\pm 0,29 \mathrm{ufc} / \mathrm{ml} \log _{10}$, es sensiblemente inferior a los reportados por los autores citados. De acuerdo a estos resultados es posible considerar que la leche estudiada presenta una muy buena calidad desde el punto de vista microbiológico.

En cuanto a la calidad de composición de leche de cabra, los valores promedio hallados fueron: materia grasa $3,58 \pm 0,69 \%$, proteína $2,71 \pm 0,07 \%$, lactosa $3,84 \pm 0,10 \%$, SNG $7,28 \pm 0,19 \%$. En Uruguay, Damián et al. (2008) consignaron valores de materia grasa, proteína y lactosa en cabras Saanen de 3,59\%, 2,84\% y 4,54\%. En Brasil, De Souza et al. (2009) presentaron los siguientes valores promedio: grasa $3,46 \%$, proteína $2,89 \%$, lactosa $4,44 \%$. Queiroga et al. (2007) reportan $3,4 \%, 2,7 \%$ y $4,1 \%$ en los mismos componentes, respectivamente, en cabras Saanen. En este trabajo se observa que los valores de materia grasa fueron levemente más altos, y los de lactosa y proteína fueron más bajos, en comparación con los de los autores citados. En Argentina, Frau et al. (2010) en cabras Saanen cruzas Anglo Nubian (75\% Saanen), pertenecientes a un rebaño con manejo extensivo, encontraron valores de 5,59\%, 3,39\%, y 4,36\% en materia grasa, proteína y lactosa. Estas diferencias evidencian la importancia de la raza y sistemas de producción en la composición de la leche. De acuerdo con Morand-Fehr et al. (2007), se registran diferencias significativas en la composición cuando la ingesta de energía proviene de sistemas de producción basados en pasturas o de sistemas intensivos con estabulación. Los sistemas de producción basados en pasturas se caracterizan por dar un alto rendimiento en el contenido de grasa láctea debido a la dieta rica en fibra. Mientras que en los sistemas intensivos, altos suministros de concentrados permiten una producción de leche rica en proteínas y relativamente baja en grasas (Morand-Fehr et al., 2007). Park et al. (2007) indican que los cambios en la composición se producen por temporadas, debido a que al final de la lactación los valores de grasa, proteína, sólidos totales y minerales se incrementan, en tanto que el contenido de lactosa decrece.

Como se especificó anteriormente, los animales utilizados en este estudio consumieron ración y praderas implantadas durante toda la lactancia. La materia grasa es el componente más variable de la leche y está muy estrechamente relacionado al tipo de alimentación. Dado que este estudio se realizó en un sistema semi extensivo (básicamente pastoril), las variaciones climáticas y de manejo afectan el tipo de alimentación y, por ende, la composición de la leche, con la materia grasa como el componente más afectado. En cuanto a la lactosa, si bien es el componente más constante de la leche, también está muy influenciado por la alimentación (principalmente relacionado a la síntesis de proteínas), por tanto las diferencias obtenidas en este trabajo podrían explicarse por el bajo porcentaje de concentrados administrado en la dieta.

En leche de cabra se encuentran los ácidos grasos de cadena corta (C4:0, C6:0, C8:0, C10:0) en una proporción tres veces mayor que en la leche de vaca (Haenlein, 2004). Según lo reportado por Páez et al. (2002), en un estudio realizado en vacas Holando alimentadas en sistemas pastoriles, la leche presentó valores de ácidos grasos de cadena corta de $2,6 \%$, muy inferior al $12,37 \%$ para leche de cabra observado en este estudio. Varios autores en distintos países han caracterizado el perfil de ácidos grasos de leche bovina. En leche 
sueca se han reportado valores para C6:0, C8:0 y C10:0 de 1,4\%, 2,7\% y 3,3\%, respectivamente (Lindmark Mansson, 2008). Valores similares se han observado en estudios realizados en vacas Holando en rebaños con distintos niveles de producción de Estados Unidos, obteniendo niveles de ácido cáprico (C10:0) de 2,79\% (Bobe et al., 2007). Ceballos et al. (2009) compararon leche de vaca y de cabra y obtuvieron valores de ácido cáprico de $3,36 \%$ en vaca y $11,07 \%$ en cabra, bajo las mismas condiciones de producción. Si bien la proporción de ácidos grasos puede ser alterada mediante factores como alimentación, animales (individuo y etapa de lactancia) y medio ambiente, hasta la fecha la mayor atención se ha centrado en los factores de alimentación, los que buscan cambios hacia un mayor porcentaje de ácidos grasos insaturados. Jensen et al. (2002) obtuvieron valores de ácidos caproico, caprílico y cáprico en los siguientes rangos: 1 a $5 \%, 1$ a $3 \%$ y 2 a $4 \%$, respectivamente. Los valores obtenidos de ácido cáprico $(8,4 \%)$ en la leche de cabra Saanen estudiada en este trabajo es de 2 a 3 veces mayor que los de leche bovina referidos en la bibliografía. Sin embargo, más allá de los cambios producidos por los factores mencionados, la leche de cabra se caracteriza por mantener mayor porcentaje de ácidos caprílico, caproico y cáprico (asociados a propiedades benéficas para la salud), en comparación con la leche bovina.

Por otro lado, es aseverado por Chacón (2005) que la leche de cabra tiene por lo general $35 \%$ de ácidos grasos de cadena mediana contra un $17 \%$ de la leche de vaca.

Según Chávez (2007), la leche de cabra presenta valores de ácidos grasos mono (MUFA) y poliinsaturados (PUFA) de $29 \%$ (dado que el $71 \%$ de los ácidos grasos de cadena larga son insaturados), los que coinciden con los valores observados en este estudio de $27,33 \%$ para leche caprina (Tabla 2). En leche bovina con alimentación exclusivamente a base de pastura se observaron valores de ácidos grasos insaturados (UFA) de 32,07\% (Páez et al., 2002), los cuales son similares y hasta sensiblemente superiores a los encontrados en este trabajo para leche caprina.

La leche de cabra presenta muy bajo contenido de ácidos grasos trans (aproximadamente 2-3\%), factor que contribuye a disminuir el colesterol denominado "malo" (Chávez, 2007). En leche de vaca se reportan valores de ácidos trans de 5,2\% (Griguol 2007). En este estudio se encontraron valores para leche de cabra de 3,04\%, que coinciden con la bibliografía citada para leche caprina (Tabla 2).

Por lo expuesto, la leche de cabra podría ser considerada más saludable en comparación con la leche de vaca, principalmente en lo relacionado a la salud cardiovascular. Esta condición estaría dada por su alto contenido de ácidos grasos de cadena corta (caproico, caprílico y cáprico) y a una más baja proporción de ácidos grasos trans que los de la leche bovina. Los ácidos grasos de cadena corta le confieren, además, la característica de ser una leche más digestible para el organismo humano, y la posicionan mejor que la leche bovina en este sentido. No obstante, no se observaron diferencias en cuanto al contenido de ácidos grasos insaturados (MUFA y PUFA) cuando se compara con leche de vacas alimentadas exclusivamente con pasturas.

Con respecto a las propiedades fisicoquímicas, en Argentina Frau et al. (2010) registraron el valor promedio de acidez de $19,52^{\circ} \mathrm{D}(16,55$ $\left.-22,63^{\circ} \mathrm{D}\right)$. Queiroga et al. (2007) analizaron leche de cabras Saanen y reportaron valores de acidez de $15,14,2$ y $16,5^{\circ} \mathrm{D}$ a los 35,85 y 135 días de lactación, respectivamente. Morgan et al. (2003) hallaron en Grecia valores de acidez de $16,17,18$ y $20^{\circ} \mathrm{D}$; en Portugal $16^{\circ} \mathrm{D}$, y en Francia de 14 y $16^{\circ} \mathrm{D}$. El resultado encontrado en este trabajo es de $14,19 \pm 0,39^{\circ} \mathrm{D}$ y es similar al citado por Queiroga et al. (2007). Existe una coincidencia con Luquet (1991) y Frau et al. (2010), quienes indican que los altos valores de acidez se encuentran asociados con altos porcentajes de caseínas. Los valores reportados por Luquet (1991) para leche de cabra son de 16 a $18^{\circ} \mathrm{D}$. A su vez, coinciden con Draksler et al. (2002), quienes afirman que la acidez de la leche se debe a la presencia de fosfatos ácidos, aminoácidos, $\mathrm{CO} 2$ y caseína en solución.

En relación a los valores de pH, Draksler et al. (2002) han encontrado variaciones de aspecto racial, pero en general la mayoría de los autores que estudiaron esta propiedad en leche de cabras Saanen observaron valores entre 6,1 y 6,7 (Park et al. 2007; Morgan et al., 2003). Este rango se podría explicar, ya que según Arnaud et al. (2009) el $\mathrm{pH}$ representa la acidez actual o verdadera de la leche, pudiendo variar durante el curso del ciclo de la lactación y bajo la influencia de la alimentación. El valor medio de $\mathrm{pH}$ obtenido en este trabajo fue de $6,66 \pm 0,06$, similar a los obtenidos por los autores citados.

En relación a la densidad de la leche de cabra, según Luquet (1991) varía entre 1,026 y 1,042 g/ml, y esta magnitud depende principalmente de dos factores: el contenido de extracto seco y la concentración de materia grasa. Ludeña et al. (2006) reportaron un valor promedio para densidad de $1,030 \mathrm{~g} / \mathrm{ml}$. La media de densidad en este estudio fue de $1,026 \pm 0,26 \mathrm{~g} / \mathrm{ml}$, valor que se encuentra dentro de los rangos reportados por los autores citados.

\section{Conclusiones}

Los resultados obtenidos de la leche caprina de raza Saanen concuerdan con otros estudios similares a nivel internacional, excepto los valores de materia grasa y lactosa que están muy relacionados a la alimentación y manejo de cada sistema productivo en particular.

Se confirmó que el rebaño de raza Saanen de este estudio posee cantidades superiores en ácidos caproico, caprílico y cáprico y bajas cantidades de ácidos grasos trans que los reportados para leche de vaca, lo cual redunda en beneficios a la salud de la leche caprina en comparación con la bovina.

\section{Reconocimientos}

Al equipo del laboratorio del Departamento de Ciencia y Tecnología de la Leche, Dr. Álvaro González, Lic. Sheila Giacaman y Lic. Karina Cal por el apoyo en los análisis de laboratorio.

A Laura Olazábal del Departamento de desarrollo de métodos analiticos del Laboratorio Tecnológico del Uruguay.

A la Prof. Adj. Carmen Silvia Gallo Muniz, encargada del área de inglés de Facultad de Veterinaria de UdelaR por la corrección del resumen.

\section{Referencias}

- AMERICAN OIL CHEMIST'S SOCIETY. Official methods and recommended practices of the AOCS. 6a ed. Urbana: AOCS, 2009. Official Method Ce 1-62.

- AMERICAN OIL CHEMIST'S SOCIETY. Official methods and recommended practices of the AOCS. 6a ed. Urbana: AOCS, 2009. Official Method Ce 2-66.

- AMERICAN OIL CHEMIST'S SOCIETY. Official methods and recommended practices of the AOCS. 4a ed., reapproved 1993.

Urbana: AOCS, 1995. Official Method Ce 1-91.

- AMERICAN PUBLIC HEALT ASSOCIATION (APHA). Compendium of methods for the microbiological examination of food. 2 ed. Washington: APHA, 1984.

\section{- AMERICAN PUBLIC HEALTH ASSOCIATION (APHA).} Compendium of Methods for Microbiological Examination of Foods. 4a.ed. Washington DC: American Public Health Association. 2001, 676p.

- ARNAUD, F.; CARRO, S.; DE LOS SANTOS, R.; GRILLE, L.; VERA, S. Acidez y prueba de estabilidad de leche y derivados. Departamento de Ciencia y Tecnología de la leche. Montevideo: Facultad de Veterinaria, Universidad de la República, 2009.

- BERGONIER, D.; CREMOUX, R.; RUPP, R.; LAGRIFFOUL, G.; BERTHEL, X. Mastitis of dairy small ruminants. En: Veterinary 
Reserch. 2009, 34:689-716.

- BOBE, G.; LINDBERG, G.; FREEMAN, A.; BEIT, D. Short communication : composition of milk protein and milk fatty acids is stable for cows differing in genetic merit for milk production. En: Journal Dairy Science. 2007, 90:3955-3960.

- BOZA, J.; SANZ, S. Aspectos nutricionales de la leche de cabra. En: Anales de la Real Academia de Ciencias Veterinarias de Andalucía Oriental. 1997, 10:109-139.

- CEBAllos, L.; MORALES, E.; DE LA TORRE ADARVE, G.; CASTRO, J.; MARTINES, L.; SANZA SAMPELAYO M.R. Composition of goat and cow milk produced under similar conditions and analyzed by identical methodology. En: Journal of Food Composition and Analysis. 2009, 22:322-329.

- CHACÓN VILLALOBOS, A. Aspectos nutricionales de la leche de cabra (Caprahircus)y sus variaciones en el proceso agroindustrial. En: Agronomía Mesoamericana. 2005, 16(2):239252.

- CHÁVEZ, M.; MARGALEF, M.; MARTÍNEZ, M. Cuantificación de lipólisis en leche caprina (Saanen) cruda y térmicamente tratada. En: ALEPRyCS. $V^{o}$ Congreso de Especialistas en Pequeños Rumiantes y Camélidos Sudamericanos, (Mendoza, 2-4 de mayo de 2007). Mendoza: ALEPRyCS, 2007.

- CONTRERAS, A.; LUENGO, C.; SÁNCHEZ, A.; CORRALES, $\mathrm{J}$.Etiología de la infección intramamaria caprina en relación con los programas de control [En línea]. [s.1.]: [s.n.], 2010. [Consulta: 6 de mayo de 2012]. Disponible en: http://www.exopol.com/seoc/ docs/1pmbcg09.pdf.

- CORDIVIOLA, C., ARIAS, R., VAAMONDE, G., LACCHINI, R., ANTONINI, A.Calidad Higiénico-Sanitaria de la leche de cabra en la cuenca de Cañuelas, Provincia de Buenos Aires. En: ALEPRyCS. $V^{o}$ Congreso de Especialistas en Pequeños Rumiantes y Camélidos Sudamericanos, (Mendoza, 2-4 de mayo de 2007). Mendoza: ALEPRyCS, 2007.

- DAMIÁN, J.P.; SACCI, I.; REGINESI, S.; DE LIMA, D.; BERMÚDEZ, J. Cheese yield, casein fractions and mayor components of milk of Saanen and Anglo Nubian dairy goats.En: Arquivo Brasileiro de Medicina Veterinaria e Zootecnia. 2008, 60:1564-1569.

- DE SOUZA, G.; RENALDI, J.; GOMES DE FARIA, C.; CASTRO, L. Composiçao e qualidade higienico-sanitária do leite de rebanhos caprinos. Produçao de Caprinos na Regiao da Mata Atlantica. Juiz de Fora, MG. En: FONSECA, J. F. da; BRUSCHI, J. H. Produção de caprinos na região da Mata Atlântica. Juiz de Fora: Embrapa Gado de Leite. Sobral: Embrapa Caprinos e Ovinos, 2009.

- DESJEUX, J.F. Valeur nutritionnelle du lait de chevre. En: Lait. 1993, 73:573-580.

- DRAKSLER, D.; NÚÑEZ, M.; GONZÁLEZ, S.; OLIVER, G. Leches de pequeños rumiantes: características generales y su microbiología. En: BARBERIS, S. Bromatología de la leche. San Luis: Hemisferio Sur, 2002. pp. 121-148.

- FRAU, S.; TOGO, J.; PECE, N.; PAZ, R.; FONT, G. Estudio comparativo de la producción y composición de leche de cabra de dos razas diferentes en la provincia de Santiago del Estero. En: Revista de la Facultad de Agronomía. 2010, 109(1):9-15.

- GARCÍA, U.; RIVERO, J.; GONZÁLES, P.; VALERO-LEAL, K.; IZQUIERDO, P.; GARCÍA, A.; COLMENARES, C. Calidad bacteriológica de la leche cruda de cabra producida en la parroquia Faría, Municipio Miranda, estado Zulia, Venezuela. En: Revista Facultad de Agronomía (LUZ). 2009, 26:59-77.

- GOMES, V.; DELLA-LIBERA, A.; PAIVA, M.; MADUREIRA,
K.; ARAÚJO, W. Effect of stage of lactation on somatic cell counts in healthy goats (Capraehircus) breed in Brazil. En: Small Ruminant Reserch. 2006, 64:30-34.

- GRIGUOL, V.; LEÓN-CAMACHO, M.; VICARIO, I. Revisión de los niveles de ácidos grasos trans encontrados en distintos tipos de alimentos. En: Grasas y Aceites. 2007, 58(1):87-98.

- HAENLEIN, G. Past, present, and future perspectives of small ruminant dairy res. En: Journal of Dairy Science. 2001, 84:20972115.

- HAENLEIN, G. Goat milk in human nutrition. En: Small Ruminant Reserch. 2004, 51:155-163.

- INTERNATIONAL DAIRY FEDERATION. (Belgium). IDF 148A: Milk enumeration of somatic cells. Brussels: International Dairy Federation, 1995.

- INTERNATIONAL DAIRY FEDERATION. IDF. (Brussels, Belgium). Milk and milk products-Guidance on sampling. FIL / IDF Standard 50C. International Dairy Federation. 1995.

- INTERNATIONAL STANDARD ORGANIZATION (Switzerland). ISO 14156/IDF 172: Milk and milk products. Extraction methods for lipids and liposoluble compounds. Geneva: ISO, 2001.

- JANDAL, J.M. Comparative aspects of goat and sheep milk. En: Small Ruminant Reserch.1996, 22:177-185.

- JENSEN, R. The composition of bovine milk lipids: January 1995 to December 2000. En: Journal Dairy Science. 2002, 85:295350.

- LARROSA, J.; KREMER, R. Leche ovina y caprina. Una nueva alternativa agroindustrial. Montevideo: Hemisferio Sur, 1990.

- LINDMARK MANSSON, H. Fatty acids in bovine milk fat. En: Food and Nutrition Research. 2008, 1821.

- LUDEÑA, F.; PERALTA, S.; ARROYO, O.; FUNG, L.; GONZALEZ, C. Caracterización físico-química y microbiológica de la leche de cabra y su conservación por el sistema lactoperoxidasa. En: Mosaico Científico. 2006, 3(1):17-27.

- LUQUET, F.M. Leche y productos lácteos. Vaca-Cabra-Oveja. Zaragoza: Acribia. 1991.

- MORAND-FEHR, P.; FEDELE, V.; DECANDIA, M.; LE FRILEUX, Y. Influence of farming and feeding systems on composition and quality of goat and sheep milk. En: Small Ruminant Reserch. 2007, 68:20-34.

- MUEHLHERR, J.; ZWEIFEL, C.; CORTL, S.; BLANCO, J.; STEPHAN, R. Microbiological quality of raw goat's and ewe's bulk-tank milk in Switzerland. En: Journal Dairy Science. 2003, 86:3849-3856.

- MORGAN, F.; MASSOURAS, T.; BARBOSA, M.; ROSEIRO, L.; RAVASCO, F.; KANDARAKIS, I.; BONNIN, V.; FISTAKORIS, M.; ANIFANTAKIS, E.; JAUBERT, G.; RAYNAL-LJUTOVAC, $\mathrm{K}$. Characteristics of goat milk collected from small and médium enterprises in Greece, Portugal and France. En: Small Ruminant Research. 2003, 47:39-49.

- PAAPE, M.; WIGGANS, G.; BANNERMAN, D.; THOMAS, D.; SANDERS, A.; CONTRERAS, A.; MORONI, P.; MILLER, R. Monitoring goat and sheep milk somatic cell counts. En: Small Ruminant Reserch. 2007, 68:114-125.

- PÁEZ, R.; GARCÍA, P.; COMERÓN, E.; ARONNA, M.; ROMERO, L.; TAVERNA, M.; PENSEL, N. Perfil de ácidos grasos en leche de vacas Holando Argentino y Jersey sometidas a dos sistemas de alimentación. En: Revista Argentina Producción Animal. 2002, 22(Suplem. 1):43-44.

- PARK, Y. Hypo-allergenic and therapeutic significance of goat milk. En: Small Ruminant Reserch. 1994, 14:151-159. 
- PARK, Y.; JUÁREZ, M.; RAMOS, M.; HAENLEIN, G. Physicochemical characteristics of goat and sheep milk. En: Small

Ruminant Reserch. 2007, 68:88-113.

- PRIDALOVÁ, H.; JANSTOVÁ, B.; CUPAKOVÁ, S.; DRACKOVÁ, M.; NAVRATILOVÁ, P.; VORLOVÁ, L. Somatic cell count in goat milk. En: Folia Veterinaria. 2009, 53(2):101105.

- PINTO, M.; VEGA Y LEON, S. Métodos de análisis de la leche y derivados. Valdivia: Universidad Austral de Chile, 1998.

- QUEIROGA, R.; COSTA, R.; BISCONTINI, T. Características microbiológicas de la leche de cabra producida en el Nordeste de Brasil [En línea]. [s.1.]: SEOC, 2002. [Consulta: 3 de mayo de 2012]. Disponible en: http://www.exopol.com/seoc/docs/vsci0opl. pdf

- QUEIROGA, R.; COSTA, R.; BISCONTINI, T.; MEDEIROS, A.; MADRUGA, M.; SCHULER, A. Influência do manejo do rebanho, das condiçoes higiénicas da ordenha e da fase de lactaçâo na composiçao química do leite de cabras Saanen. En: Revista Brasilera Zootecnia. 2007, 36(2):430-437.

- Reglamento bromatológico nacional decreto 315/1994 de fecha 05/07/1994 : anotado y concordado con apéndice normativo. Montevideo: MSP, 1994. pp. 143-155.

- RIBEIRO, A.; RIBEIRO, S. Specialty products made from goat milk. En: Small Ruminant Reserch. 2010, 89:225-233.

- ROBERTSON, N.; MULLER, C. Somatic cell count in goat's milk as an indication of mastitis [En línea]. En: SA-ANIM SCI, 2005, v. 6. [Consulta 8 de mayo de 2012]. Disponible en: http://www.sasas. co.za/sites/sasas.co.za/files/RobertsonAPop05.pdf

- SANZ, S.; FERNÁNDEZ, J.; DE LA TORRE, G.; RAMOS, E.; CARMONA, F.; BOZA, J. Calidad de la leche de los pequeños rumiantes. En: Anales de la Real Academia de Ciencias Veterinarias de Andalucía Oriental. 2003, 16:155-166.

- SCHMIDT, V.; GOTTARDI, C.; MURICY, R.; CARDOSO, M. Qualidade higiênica de leite caprino por contagem de coliformes e estafilococos. En: Ciência Rural. 2008, 38(3):743-748.

- ZWEIFEL, C.; MUEHLHERR, J.; RING, M.; STEPHAN, R. Influence of different factors in milk production on standard plate count of raw small ruminant's bulk-tank milk in Switzerland. En: Small Ruminant Reserch. 2005, 58:63-70. 